\title{
Structural Symmetry of DNA Nucleotides and Steroid Hormones
}

\author{
Charles D. Schaper, Ph.D. \\ Transfer Devices, Inc. \\ Union City, CA 94587 USA \\ text: $510-378-8260$ \\ Corresponding author: Charles D. Schaper, Ph.D. \\ cschaper@transferdevices.com
}

March 16, 2020 


\begin{abstract}
DNA is comprised of important structural characteristics, which include the complementary base pairs of adenine-thymine (A-T) and cytosine-guanine (C-G) that serve to initiate and code for transcription and translation into amino acids. Recently, structural analysis of DNA performed in this lab indicated that each DNA nucleotide complementary base pair is in perfect correspondence with the structure of the steroid molecule and steroid hormones. Here, detailed structural analysis and illustrations are presented to clearly support and extend this fundamental finding. The structural illustrations indicate that the DNA Nucleotide base pairs can achieve perfect alignment with steroid hormones, such that each of its functional groups can be assigned a purpose for binding, stabilization, and transcription regulation. The relation of the missing third hydrogen bond for A-T and T-A, relative to the three hydrogen bonds of C-G and G-C, is clearly shown to be found through its coupling with the class of corticosteroids like cortisol that have an oxygen group perfectly positioned for interaction with the available functional group of thymine. Thus the intermolecular coupling by hydrogen bonding of Cortisol-Thymine produces a strong complex. Moreover, the structural analysis of the end group couplings to an ionic linkage element, $\mathrm{Ca}^{2+}$ or $\mathrm{Mg}^{2+}$, demonstrate interaction with both the DNA phosphates as well as the oxygen element within the sugar. The relationships confirm a structural association of cortisol-like steroid hormones with A-T and T-A and a structural association of testosterone-like steroid hormones for G-C and C-G. Synthetic steroids are assessed, including prednisolone and dexamethasone, to indicate consistency of the functional group interactions with the DNA base pairs, phosphate, and sugar groups to support and confirm direct binding and structural correspondence of steroid hormones to DNA Nucleotides.
\end{abstract}




\section{Introduction}

DNA is comprised of the complementary base pairs Adenine-Thymine (A-T) and CytosineGuanine (C-G), organized along a phosphate-oxygen-carbon chain, connected with a carbonoxygen sugar, in which the base pairs couple through hydrogen bonding [1]. The A-T pairing has two hydrogen bonds, while the $\mathrm{C}-\mathrm{G}$ pairing has three hydrogen bonds, although the Thymine molecule has an unpaired functional group. The base pairs encode protein structures trough a continuous set of base-pair elements, preceded by initiation sites, which are also comprised of nucleotide base pairs, which when activated enable the separation of two DNA strands to allow the activation of RNA polymerase to perform gene transcription $[2]$.

Gene expression is a primary objective of DNA. The mechanisms to enable gene expression have been hypothesized to involve the interaction of protein complexes with the DNA nucleotides to induce strand separation and RNA polymerase incorporation for mRNA transcription $[3,4,5]$. Further, the hypothesis incorporates the various loops, turns, major grooves, minor grooves, and hairpin secondary structures of DNA as locations as available but necessary to achieve transcription $[6,7,8,9]$. In addition, the process of DNA transcription is hypothesized to involve a number of protein transcription, activators and co-factors transcription $[10,11,12,13,14]$. Further, the interaction of steroid hormones is considered to be involved as simply a receptor process in which the protein element transporting the steroid hormone to the nucleus binds to DNA through an allosteric effect to participate in the transcription process $[15,16,17,18,19]$.

However, in my recent work [20], a much simpler hypothesis for DNA transcription was developed in which the central tenet is that steroid hormones directly bind to DNA nucleotides in order to enable and initiate DNA transcription. A one-to-one structural correspondence was established between the steroid molecule, steroid hormone and DNA nucleotide pairing, coupled through an ionic mechanism. This induces localized stabilization of the DNA molecule, whose energy must also be balanced locally through destabilization of the internal hydrogen bonds connecting the nucleotides, thereby inducing gene transcription through strand separation and RNA polymerase incorporation. In this work, the structural correspondence is examined further, using illustrations and analysis that precisely relate the correspondence and interaction of steroid hormones and DNA nucleotides.

\section{Results}

The first results associating the steroid molecules and steroid hormones with DNA Nucleotides were presented in my recent work [20] and in this section, illustrations are used to unequivocally document the relation. The section is organized by first presenting the illustrations for structural symmetry of DNA nucleotides with steroid molecules and steroid hormones. The coupling points including ionic and intermolecular hydrogen bonding are 
examined. The regulatory elements are also described such that each element comprising the nucleotide and the steroid hormone are associated with form and function.

\subsection{Structural Correspondence of Steroid Molecule and DNA Nucleotides}

In this subsection, a detailed illustrative analysis is presented of the inherent steroid structure embedded within each DNA Nucleotide complementary base pairing. In Figure 1, the front side of the DNA Nucleotide pair is indicated for A-T, T-A, C-G, and G-C. In Figure 1, the back side of the same DNA Nucleotide pairs is also indicated. Both also show the phosphate-sugar backbone connecting the DNA Nucleotides. This arrangement is shown for illustrative and explanatory purposes. The arrangement can be envisioned as the DNA Nucleotide structure printed on paper, and then viewing it from the front and back side of the paper. The hydrogen-bond is noted in the illustration, in which there are three hydrogen bonds for the $\mathrm{C}-\mathrm{G}$ and $\mathrm{G}-\mathrm{C}$ pairings but only two hydrogen bonds for the adenine-thymine A-T and T-A pairs. It is interesting also, that while there are only two hydrogen bonds for A-T, there exists anyway one functional group on thymine that does not have hydrogen bonding.
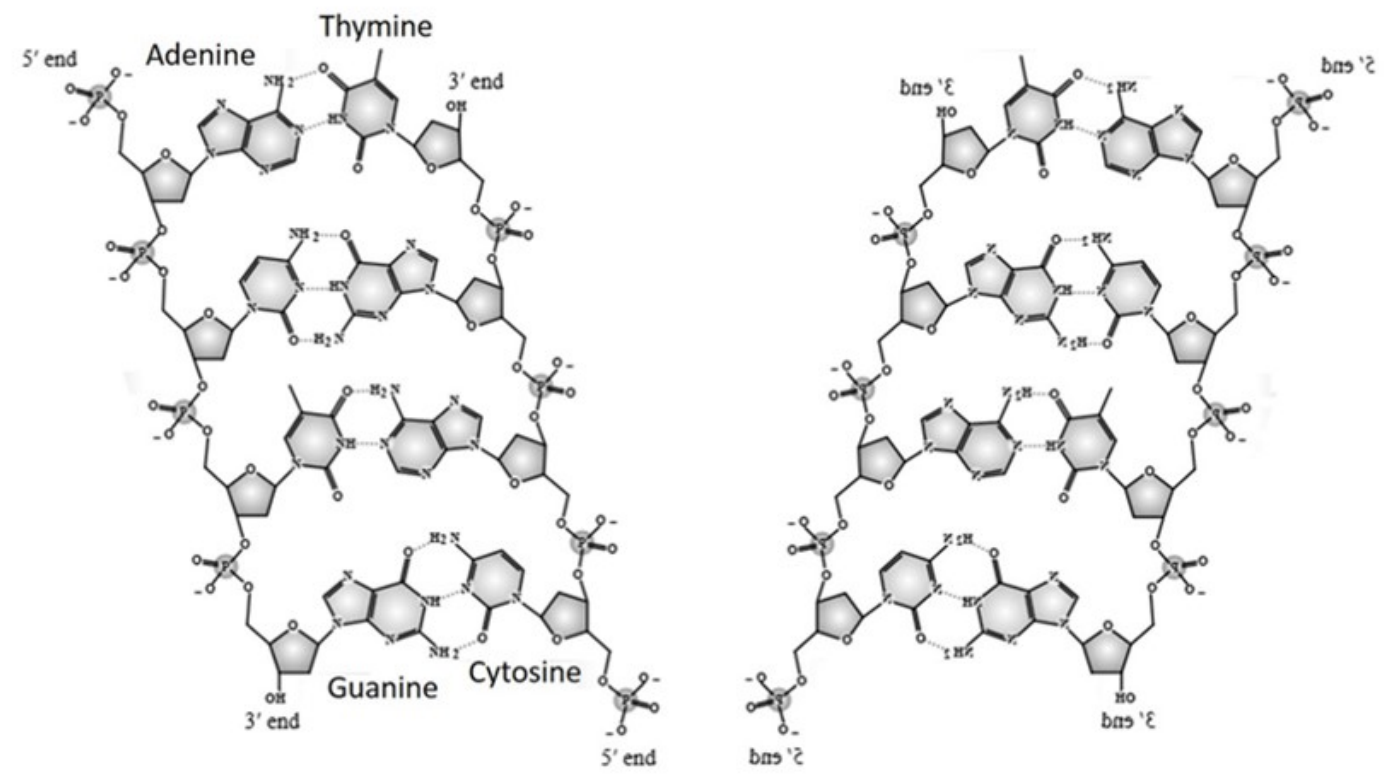

Figure 1: The structure of the four DNA nucleotide pairs, $A-T, T-A, C-G$, and $G$-C, indicating both the front side and the back side of the DNA molecule. The back side, which would be equivalent to that which would be seen on the back of a printed page with the molecule on the front, is obtained by flipping the molecule horizontally. Both orientations are important to understanding the structure relations. 
Now, a rather extraordinary result is presented in Figure . By connecting the oxygen, nitrogen, and carbon elements in a particular manner, it is possible to trace a four-ring structure into each of the nucleotide pairs. Subsequently, by extracting and rotating the four-ring structure, a perfect correspondence among each of the four results. Moreover, remarkably, each four-ring structure corresponds to that of a steroid molecule. In addition, it is also interesting to note that the four-ring structure of the steroid molecule is produced exactly on the front side for the A-T and G-C configuration. However, for the reverse T-A and C-G pairings, the four-ring structure of the steroid molecule is formed on the back side of the base pairs. It is also noted that there is a stretching of one of the rings due to the hydrogen bond length distance being longer than the covalent bonds comprising the other rings.

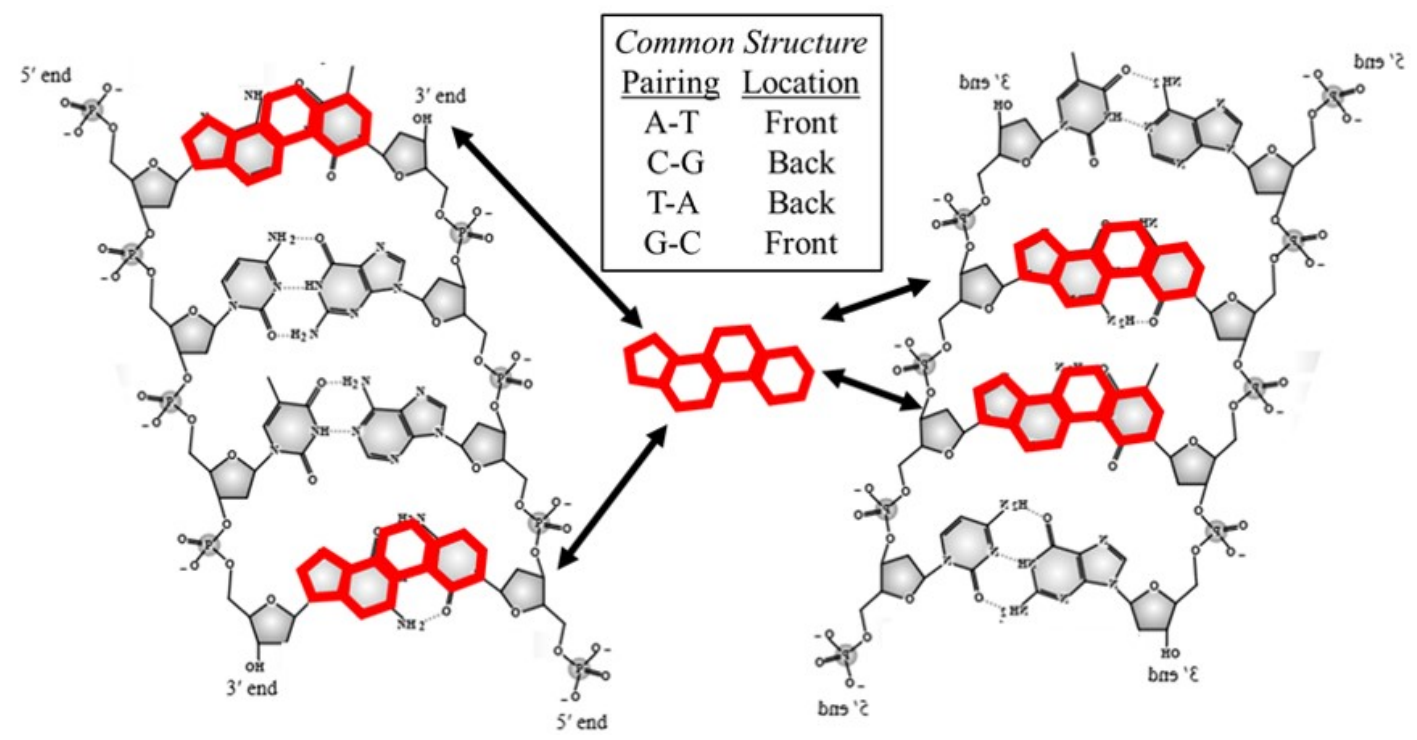

Figure 2: The red outline shows the arrangement of a four-ring structure common to each DNA Nucleotide complementary base pairs. Each DNA Nucleotide complementary base pairs possess the exact same structure. Moreover, the shape of the four-ring structure corresponds to that of a steroid molecule. Furthermore, for this steroid molecule structure, the $A-T$ configuration aligns on the front side and the T-A configuration aligns on the back side; the $G-C$ configuration aligns on the front side and the $C$ - $G$ configuration aligns on the backside.

\subsection{Structural Symmetry of Steroid Hormones and DNA Nucleotides}

In this section, the structures of DNA Nucleotide pairs are related to the steroid hormones. In Figures 3(a) and 3(b), the molecular structures of cortisol and testosterone are indicated. The cortisol molecule is representative of the steroid hormones that have an 
oxygen group in the middle of the molecule, in addition to the oxygen groups on each end. A related molecule would be aldosterone, for example, in addition to others. The testosterone molecule is selected because the functional groups are arranged only at the ends of the molecule and thus there is no functional group in the middle. Related steroid hormones would be progesterone and estrogen, among others.

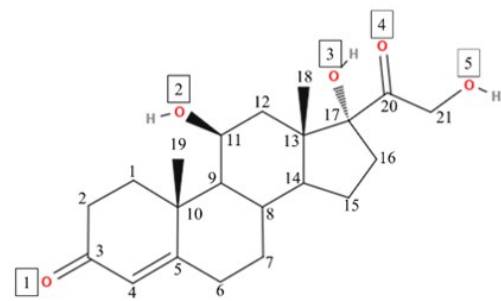

(a) Cortisol

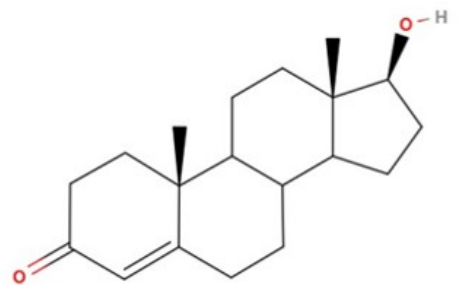

(b) Testosterone

Figure 3: The (a) cortisol molecule is notable in that there is a mid-sectional oxygen group O2 at $C 11$, in addition to the end groups at $C 3$ and $C 1 \%$. In (b), testosterone, only contains the end-groups placed in a similar position to that of cortisol, and does not contain a mid-section functional group. This will be important in the analysis

In Figures 4(a) and 4(b), structural symmetry is demonstrated between steroid hormones and DNA Nucleotides. In fact, there is a perfect correspondence. To achieve the match, the unfilled hydrogen bond of A-T and T-A is filled with the middle oxygen group of cortisol. Since G-C and C-G already have three hydrogen bonds, testosterone is matched to the structure. Thus, for each steroid hormone-DNA Nucleotide complex, a full set of three hydrogen bonds results. In order to get the result to work, a matching of the steroid hormone had to be performed on the front and on the back of the DNA Nucleotide pairs. Moreover, for the backside orientation, the representative steroid hormone was flipped vertically and flipped horizontally. Thus, due to the twice flipping, the orientation of the side-groups of the steroid hormones relative to DNA will be the same whether on the front side or the back side, including of course the hydrogen bond and the end-groups. Further, with both types of steroid hormones, the oxygen end groups are positioned towards the phosphate backbone. In addition, the cortisol molecule contains additional oxygen structures on one of its end group that are positioned in association with the sugar. Further, although not shown here, the arrangement holds even in the rare event that thymine is rotated about its single bond connection to the sugar, but with the exception that that the steroid hormone aligns on the back side of $\mathrm{A}-\mathrm{T}$, thus indicating a source of potential new functionality. Further, there is one oxygen on the end of the cortisol molecule that is positioned away and apparently independent of the phosphate-sugar backbone. Thus, there is a code of orientation (front/back) and class (presence/absence of hydrogen bonding to Thymine), which will be the subject of a future study. 


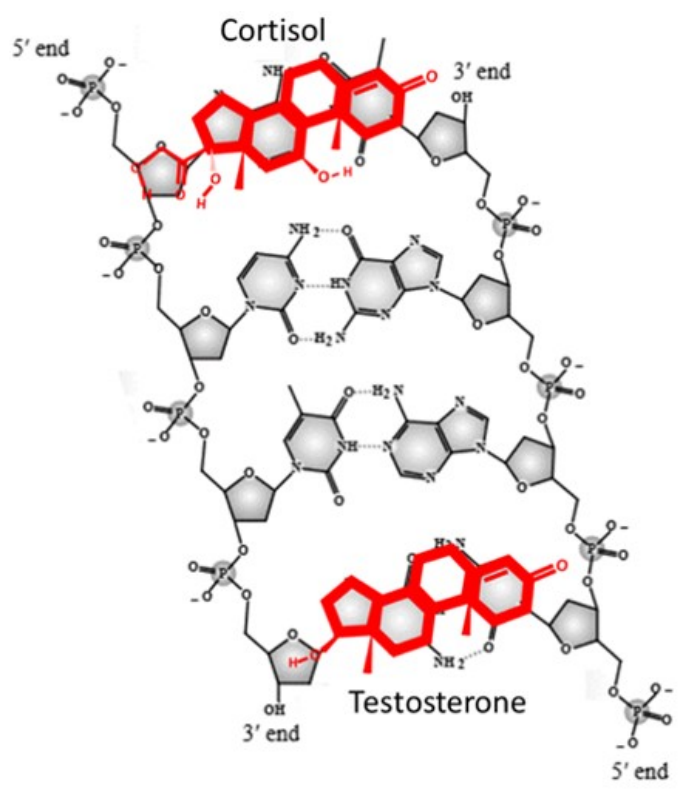

(a) Front with Steroid Hormone

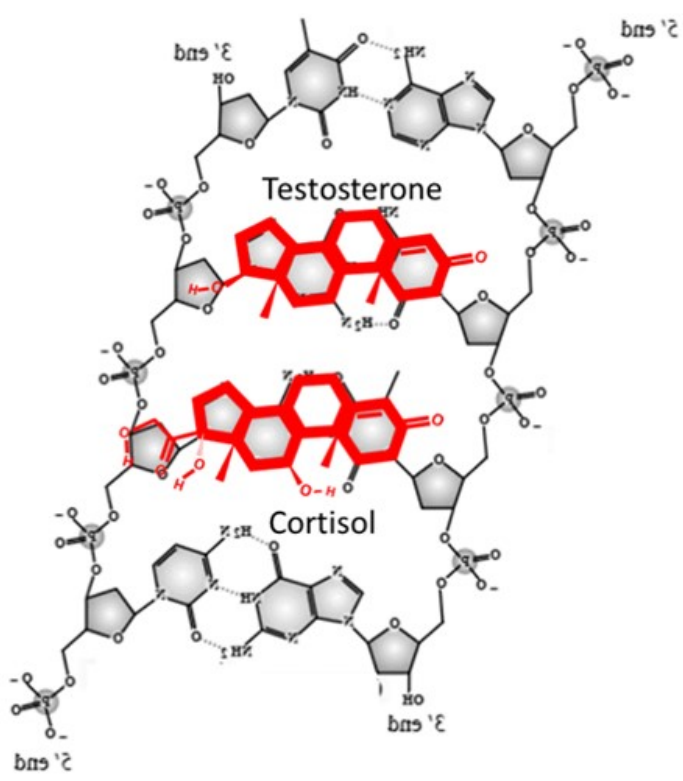

(b) Back with Twice Flipped Hormone

Figure 4: This illustration depicts the correspondence of the cortisol steroid hormone with the A-T and $T$ - $A$ pairs and the testosterone steroid hormone with the $C$ - $G$ and $G$-C pairs, in order for there to be three hydrogen bonds for each of the DNA nucleotide arrangements. It is noted that there is a (a) front-side and (b) back-side orientation. To get the backside of the molecule to match, the hormone had to be flipped both in the horizontal and the vertical directions.

\subsection{Correspondence of Steroid Hormone End Groups to Phosphate-Sugar Backbone}

In the following subsections, molecular models are utilized to support the relationship of the steroid hormone binding and symmetry with DNA nucleotide pairs through an ionic coupling agent, $\mathrm{Ca}^{2+}$ or $\mathrm{Mg}^{2+}$ ion, as presented originally in [20]. The relation of the endgroups to the phosphate sugar backbone is presented in Figure 5 for the steroid hormone cortisol. The results associated with cortisol will be like those with testosterone in terms of one of the end groups, a ketone, but the other group, is a bit different since additional groups on cortisol are available for linkage. In the case of testosterone, one end has a ketone group facing one end, with the other end utilizing a hydroxyl group. For cortisol, one end has a ketone group as with cortisol, however the other end is far more complex.

The end group for one side of the cortisol molecule is depicted in Figure 7. The ionic coupling is seen to involve a significant number of charged elements, including the two oxygen groups associated with the phosphate backbone, and even the oxygen group of the connecting sugar group. The ketone group of the cortisol end is connected to the positively 


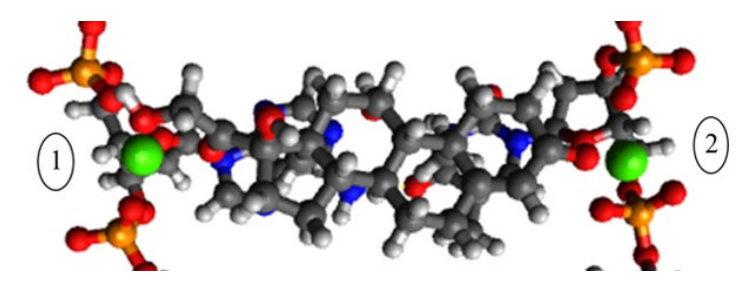

Figure 5: Molecular model of the relationship of the ionic coupling points (shown in green denoted as 1 and 2) to the phosphate-sugar backbone of the DNA nucleotide pairs and the associated steroid molecule, cortisol in this case.

charged ionic coupling agent, as is the hydroxyl group. With an appropriate movement of the hydroxyl, hydrogen bonding with one of the phosphate oxygen ions becomes possible as well. The positively charged ionic coupling element is positioned symmetrically with respect to the adjacent phosphate groups.

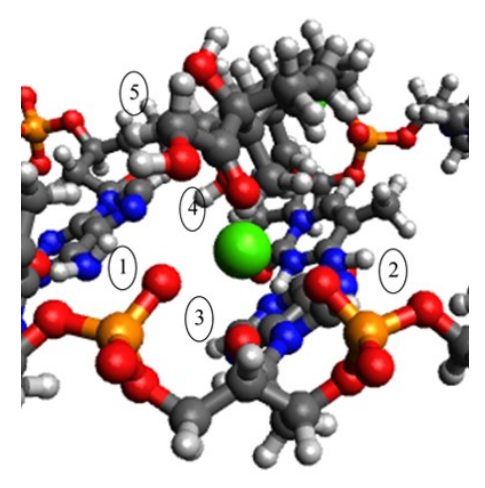

(a) Ion/Steroid/DNA coupling

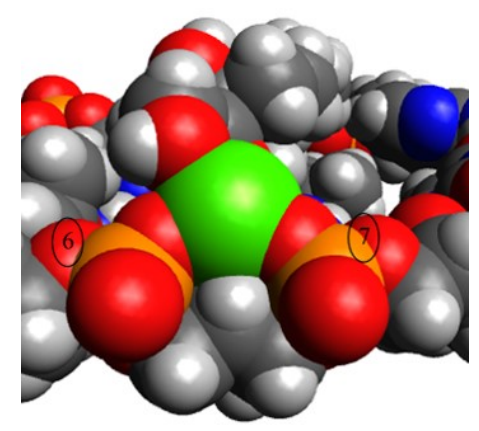

(b) Van der Waals spheres

Figure 6: At the coupling point end (a) for cortisol C17, C20, C21, and O3, O4, O5, there includes phosphate oxygen groupings (1) and (2), as well as the oxygen (3) on the sugar, all of which interact with the ionic coupling point. From the steroid complex, points (4) and (5) interact with the ionic point, $\mathrm{Ca}^{2+}$, or other ionic material. (b) the van der Waals representation indicates the phosphate grouping of the DNA nucleotide (6) and (7) overlapping the ion, $\mathrm{Ca}^{2+}$.

At the other end of the cortisol steroid hormone, the ionic coupling point is examined in Figure 7(a). It is seen that there is coupling at the two adjacent phosphate groups as well as the oxygen on the sugar group, with the steroid connection occurring at the ketone group. The structure is slightly non-symmetric with respect to the phosphate interaction with the ionic coupling element. 


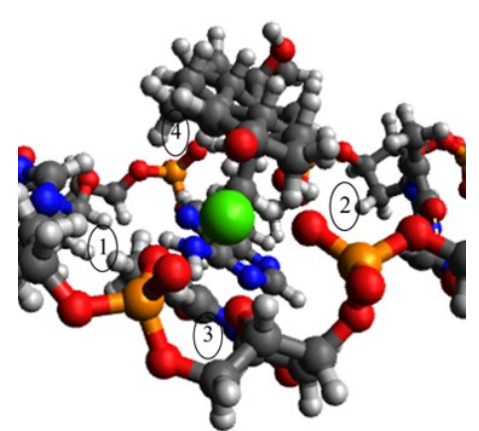

(a) Ion/Steroid/DNA coupling

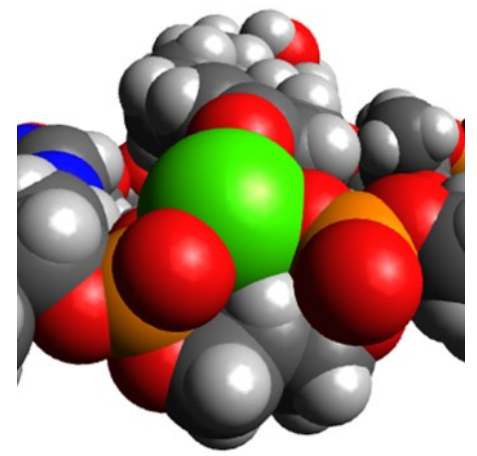

(b) Van der Waals spheres

Figure 7: For the steroid hormone cortisol groups $C 3$ and $01,(a)$ the coupling points include the phosphate oxygen groups (1) and (2), and the sugar oxygen group (3), along with the steroid cortisol oxygen group (4). In (b) the van der Waals model is depicted showing slight asymmetry of the coupling points to the ion, $\mathrm{Ca}^{2+}$.

\subsection{Hydrogen Bonding of Cortisol to Thymine}

The connection of the hydroxyl group on the C-ring of cortisol to the available ketone group on the Thymine molecule is very important. It achieves an intermolecular connection with the steroid molecule, which together with the binding to the ionic group at both ends of the steroid molecule, achieves a strong connection. Thus, the A-T pairing would be expected to be prevalent in regulatory elements initiating gene transcription through localized stabilization of the DNA molecule. To assist with the stabilization process, there is a methyl group at the intersection of the $\mathrm{A}$ and $\mathrm{B}$ rings that projects in the same direction as the hydroxyl group. In a structural relation it will prevent the hydroxyl group from getting to close to the ketone group, and act in some ways as a spacer in forming the full steroid-ion-DNA complex. In Figure 8, the hydrogen bond coupling is indicated between the steroid hormone and the Thymine base.

\subsection{Regulatory Transcription Groups of Steroid Hormones}

In addition to the functional groups for attachment to the DNA molecule, the steroid hormones also contain additional oxygen groups that are not affiliated with the attachment purpose. There are five functional groups of cortisol, three of which are accounted for in terms of the intermolecular binding to the DNA molecule. There is one group, labelled as (2) in Figure 10, of a hydroxyl that is available for binding to the ionic coupling point, but is available for outward connection to the external environment. In particular, there is a second hydroxyl group that is not affiliated with the ionic coupling point, which is 


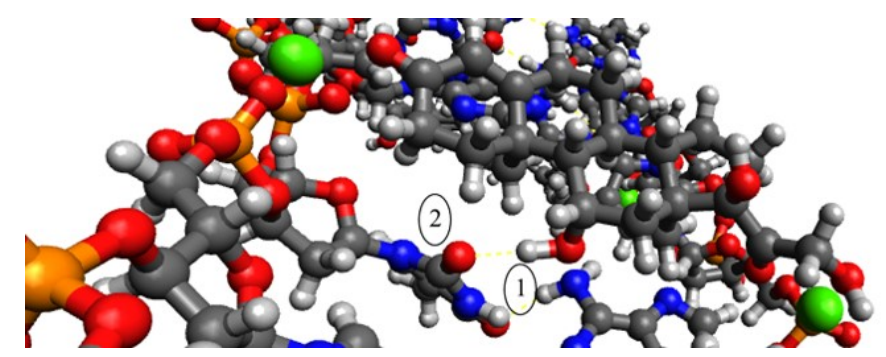

Figure 8: The relation of the Thymine oxygen group with the hydroxyl group of O2 on C11 of cortisol with a spacing less than $2 \AA$.

immediately available for coupling to the external environment. It is considered that this group interacts with the RNA polymerase, which will also aid with the stabilization of the molecule.

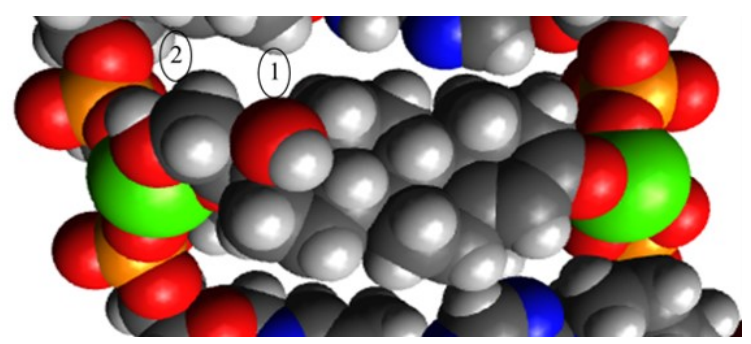

Figure 9: There are two potential gene regulatory elements associated with the steroid hormone cortisol when connected to the DNA strands, as labelled as (1) and (2), which are O2 on C11 and $\mathrm{O} 3$ at C17. O2 is not interacting with the ionic coupling points, and is thus fully available to interact with RNA polymerase.

This coupling is further examined in evaluating the electrostatic potential of Figure 10. It indicates a positive charge relative to the phosphate backbone. Thus, the coupling of gene transcription regulatory elements can serve as a differentiated point of interaction.

\subsection{Regulatory Groups of Synthetic Steroid Therapeutics}

The synthetic steroid therapeutics have been studied in terms of their effectiveness with respect to slight modifications in their molecular construction, and thus aid in evaluating the hypothesis of the steroid hormones directly binding to DNA. In Figure 11, three molecules are studied in this subsection are shown. The difference between prednisone and prednisolone is just one hydroxyl group instead of a ketone group. And the difference between prednisolone and dexamethasone is essentially just the addition of a fluoride element. All three groups have a double bond at the $\mathrm{C} 1-\mathrm{C} 2$ position, which is different than cortisol. All three molecules, as well as cortisol, have different effectiveness, with an equivalent dose of 


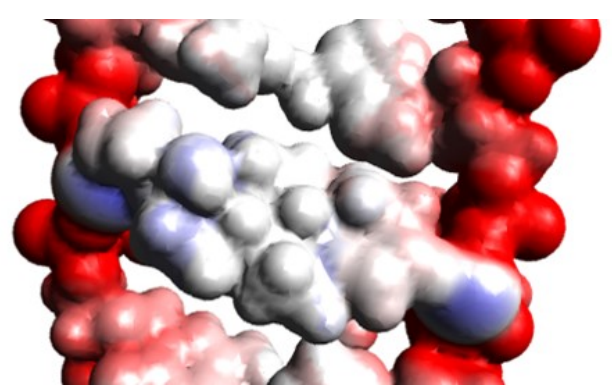

Figure 10: The electrostatic potential of the cortisol steroid hormone in conjunction with the ionic coupling point and phosphate-sugar backbone. It is noted that there is the positive charge associated with the ionic points which can serve as a regulatory indicator point for connection with the RNA polymerase structure.

approximately 25:5:1 for cortisol:prednisolone:dexamethasone with dexamethasone as the most potent, and the developments of this paper can be utilized to provide an explanation for this relation and difference in effectiveness.

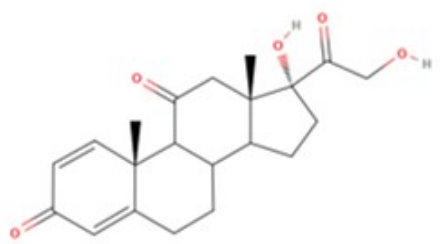

(a) Prednisone

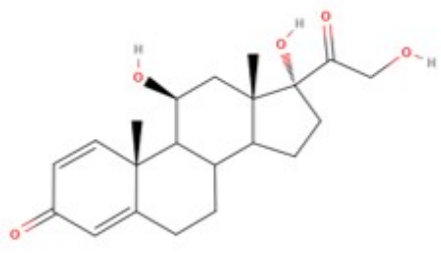

(b) Prednisolone

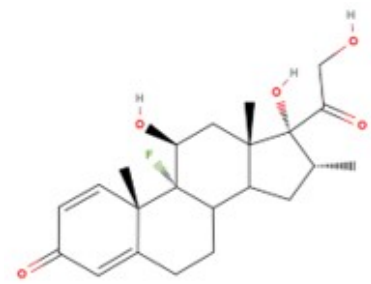

(c) Dexamethasone

Figure 11: Synthetic steroids which show close relation to cortisol are utilized with varying effects. (a) Prednisone differs from cortisol with a double bond at C1-C2 and a ketone at C11, O2, but is inactive; (b) Prednisolone differs by just the double bond at C1-C2, but is several times more effective than cortisol; and (c) differs from Prednisolone substantially by the fluoride group at $C 9$.

By examining the difference between prednisone and prednisolone, the importance of the hydroxyl group in achieving hydrogen bonding is noted. Prednisone is not effective at all, and must be converted to prednisolone before it is useful. Clearly, it indicates the intermolecular coupling of the hydrogen bond with Thymine is important and necessary. This is further amplified by noting the $\mathrm{C} 1-\mathrm{C} 2$ double bond indicates that there is stronger hydrogen bonding than cortisol since resonance stabilization can take place, and the oxygen group of Thymine will become more nucleophilic since the electrons of the double bond can more fully establish with the oxygen group that interacts with the steroid hormone. In Figure 12, the molecule of dexamethasone is indicated with respect to its correspondence 
to A-T, where it is seen that it is much like prednisolone except for the fluoride group. The activity of dexamethasone is greater than that of prednisolone. Thus, because the fluoride group is facing the back of the molecule, away from the intermolecular hydrogen bond, and away from the ionic coupling. Therefore, it has the potential to act as an additional regulatory element.

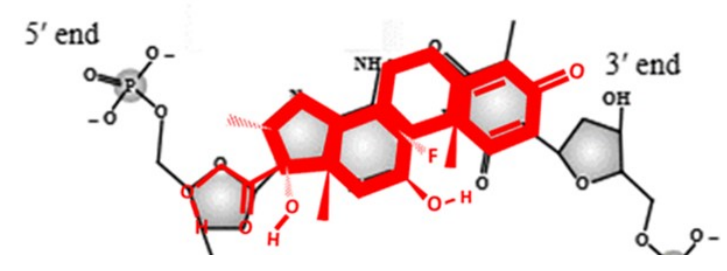

Figure 12: For dexamethasone, the relationship of fluorine can be seen with respect to the A-T nucleotides, but is positioned on the back of the molecule, as with the hydroxyl group at C17.

In Figure 13, the backside of the dexamethasone molecule is indicated, in which it is shown that the fluoride element is facing the RNA polymerase activity, similar to the two hydroxyl groups. Thus, there is an additional element for interaction and stabilization with the external environment, and thus one would expect dexamethasone to have additional functionality and activity relative to prednisolone.

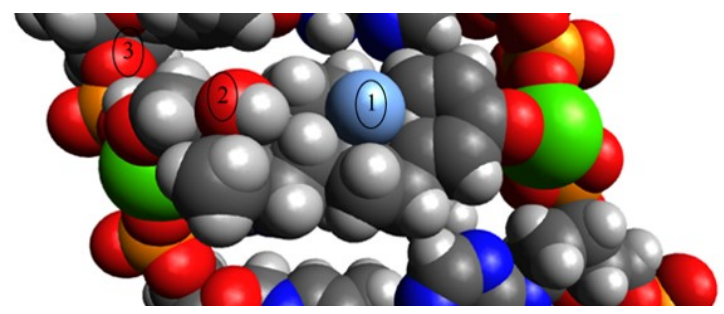

Figure 13: The position of the regulatory items for dexamethasone as binded to the A-T nucleotide, comprising (1), fluoride, (2), hydroxyl, and (3), hydroxyl. There is significant interaction potential for the RNA polymerase because of the fluoride ion, which is in addition to the normative hydroxyl groups.

\section{7 lon connection point}

The developments presented in this research article indicated calcium ion $\mathrm{Ca}^{2+}$ as the critical coupling point for the steroid hormone and the DNA nucleotides. As noted in [20], there are several reasons for this implementation, but it is noted that magnesium ion $\mathrm{Mg}^{2+}$ can also be utilized, because it results in a symmetric coupling with adjacent phosphate groups, as indicated in Figure 14. For this configuration, the distances are tighter: for example, the distance from the phosphate group to the ion at the C17 side is $3.213 \AA$ and 
$3.176 \AA$ for magnesium versus $3.469 \AA$ and $3.524 \AA$ for calcium. Also, the energy of the structure is favorable for $\mathrm{Mg}^{2+}$ than that of $\mathrm{Ca}^{2+},-13,080 \mathrm{~kJ} / \mathrm{mol}$ for magnesium versus $-11,755 \mathrm{~kJ} / \mathrm{mol}$ for calcium. The electrostatic potential is different for $\mathrm{Mg}^{2+}$ however, with a slightly more negative charge due to the compressing of the phosphate ions, and less differentiated than $\mathrm{Ca}^{2+}$ relative to the DNA phosphate backbone, thus less selectivity with respect to potential electrostatic guidance.

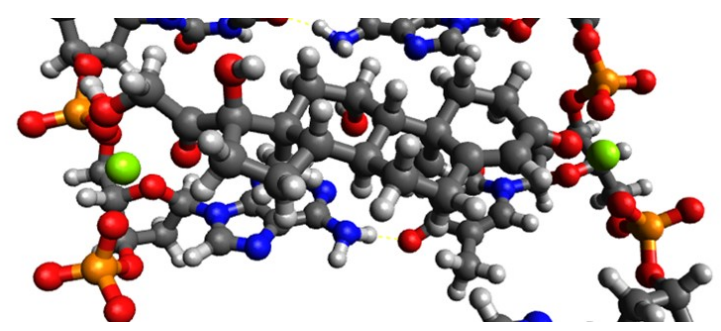

Figure 14: The ionic coupling point is very important to the stabilization of the DNA molecule, which is necessary in order to enable gene transcription. The two candidates are $\mathrm{Ca}^{2+}$ and $\mathrm{Mg}^{2+}$ as indicated. The dimensions of the interactions with the steroid and DNA complexes are approximately equivalent

\section{Discussion}

The inherent structure within each of the DNA Nucleotide complementary base pairs, is a remarkable finding, which was achieved of a steroid molecule by connecting the carbon, oxygen and nitrogen elements in a particular manner. Thus, it is the case that the steroid molecule is the structural basis of DNA. The purine and pyrimidine bases account for the three of the four rings of the steroid molecule, and their coordination accounts for the fourth.

From a developmental or evolutionary point of view, it makes sense that the steroid hormone and the DNA bases would share the same structural basis. The steroid hormone can therefore align and trigger the DNA molecule as its target to produce proteins, and thus each are developed simultaneously from the same structure. If proteins triggered DNA molecules, it creates the problem of which one came first, the protein molecule which would be needed to trigger DNA production; or the DNA molecule, which would require a protein to be of use. By having the same structure as the basis for the trigger and target, as is the case for the steroid hormone - DNA Nucleotide Pairs, this problem is resolved.

Moreover, the structural organization of the steroid molecule inherent to each DNA nucleotide pairs extends precisely to the functional groups of the steroid hormones. Each of the functional groups comprising the steroid hormones has a purpose in its interaction with the DNA molecule, and its subsequent application for gene transcription. The steroid hormone contains multiple capabilities: (a) the entire ring structure is to stabilize the DNA 
molecule; (b) the end groups and mid-sectional functional group is to bind the steroid hormone to the DNA Nucleotides; (c) there are spacing elements to direct its association; (d) there are directionality markers to interact with RNA polymerase. This information is useful in drug design as there is already confirmation of this concept of structural symmetry which can be applied to synthetic steroids, such as prednisolone and dexamethasone, to indicate the reason for the effectiveness of these molecules in comparison to cortisol.

Furthermore, the location of the missing third hydrogen bond correspondence is perfect in terms of the location of the mid-section hydroxyl group of cortisol-like molecules with the unmatched functional group of Thymine. This result signifies strong evidence that steroid hormones interact directly with the DNA molecule, as it is beyond coincidental. Previously, the A-T and T-A pairs were considered as "weak", but when paired with the steroid hormone, it leads to a strong complex, because of the intermolecular actions.

The alternative hypothesis that DNA interacts with protein, that is a protein-DNA hypothesis, to affect gene transcription needs to evaluated in light of these results. The initiation sites are only nine base pairs, and the potential interaction with protein molecules seems unlikely to contain sufficient disruptive energy to explain the site of gene transcription. Moreover, DNA is quite stable as it is known to survive for millions of years, and requires $95^{\circ} \mathrm{C}$ to denature. It seems unlikely that interactions with several amino acid side residues would disrupt DNA enough in vivo with sufficient precision to produce gene transcription, as is the case with the protein-DNA hypothesis.

The gene regulatory action of the steroid hormone was noted. The steroid hormone thus plays a role in stabilizing the DNA structure, in order to destabilize locally to induce transcription. As well, the steroid hormone provides guide posts on the back from which RNA polymerase may attach and achieve the transcription process. The work has successfully identified a purpose for each of the elements comprising the steroid hormone, including its end groups for attachment, interior group for hydrogen bonding and selectivity.

A fundamental result in the structural form and functionality of DNA has been shown. It likely indicates the profound result that the steroid hormones bind directly to DNA to enable gene transcription, and perhaps are the only manner in which gene transcription can take place. The molecular correspondence between the steroid hormones and DNA nucleotides is perfect in structure and symmetry.

\section{Methods}

- Molecular Modeling: the software program Avogadro was used to build three dimensional molecular models and to evaluate the interaction of steroid hormones with DNA nucleotide sequences. Cortisol was selected as the steroid hormone to utilize in the simulations because it has the appropriately positioned mid-molecule oxygen group, because of its functional groups at each end, because of its importance in physiology, and because of prior work in understanding its relationship with the lig- 
and binding domain. The force field MMFF94 [21] was used within the Avogadro software program to optimize the position of the steroid hormone with respect to the DNA nucleotides.

- Interaction of Steroid Hormone and DNA Nucleotide Sequence: Eight alternating pairs of A-T nucleotides were prepared using the software program, and the first association was made at the Nucelotide 5 position, and after stabilization, the second association was made at Nucleotide 2 position on the opposite side. The optimization routine was utilized to determine the lowest energy state. Using previously described methods [22], the calcium and magnesium ions were coupled to the DNA nucleotides with the steroid hormone. 


\section{Bibliography}

[1] J. D. Watson and F. H. Crick, "Molecular Structure of Nucleic Acids," Nature, vol. 171, no. 4356, pp. 737-738, 1953.

[2] J. Watson, T. Baker, S. Bell, A. Gann, M. Levine, and R. Losick, Molecular Biology of the Gene. Cold Spring Harbor Laboratory Press, 2014.

[3] T. Kokubo, D.-W. Gong, R. G. Roeder, M. Horikoshi, and Y. Nakatani, "The drosophila 110-kda transcription factor tfiid subunit directly interacts with the nterminal region of the 230-kda subunit," Proceedings of the National Academy of Sciences, vol. 90, no. 13, pp. 5896-5900, 1993.

[4] N. C. Smith and J. M. Matthews, "Mechanisms of dna-binding specificity and functional gene regulation by transcription factors," Current opinion in structural biology, vol. 38 , pp. 68-74, 2016.

[5] P. Martin-Malpartida, M. Batet, Z. Kaczmarska, R. Freier, T. Gomes, E. Aragón, Y. Zou, Q. Wang, Q. Xi, L. Ruiz et al., "Structural basis for genome wide recognition of 5-bp gc motifs by smad transcription factors," Nature communications, vol. 8, no. 1, pp. 1-15, 2017.

[6] T. Q. Trinh and R. R. Sinden, "Preferential dna secondary structure mutagenesis in the lagging strand of replication in e. coli," Nature, vol. 352, no. 6335, pp. 544-547, 1991.

[7] O. Miura, T. Ogake, H. Yoneyama, Y. Kikuchi, and T. Ohyama, "A strong structural correlation between short inverted repeat sequences and the polyadenylation signal in yeast and nucleosome exclusion by these inverted repeats," Current genetics, vol. 65, no. 2, pp. 575-590, 2019.

[8] J. H. White and W. R. Bauer, "Superhelical dna with local substructures: a generalization of the topological constraint in terms of the intersection number and the ladder-like correspondence surface," Journal of molecular biology, vol. 195, no. 1, pp. 205-213, 1987.

[9] E. Paleček, "Local supercoil-stabilized dna structure," Critical reviews in biochemistry and molecular biology, vol. 26, no. 2, pp. 151-226, 1991.

[10] S. Inukai, K. H. Kock, and M. L. Bulyk, "Transcription factor-dna binding: beyond binding site motifs," Current opinion in genetics $\mathcal{E}$ development, vol. 43, pp. 110-119, 2017. 
[11] C. Chronis, P. Fiziev, B. Papp, S. Butz, G. Bonora, S. Sabri, J. Ernst, and K. Plath, "Cooperative binding of transcription factors orchestrates reprogramming," Cell, vol. 168, no. 3, pp. 442-459, 2017.

[12] M. P. Stemmler, R. L. Eccles, S. Brabletz, and T. Brabletz, "Non-redundant functions of emt transcription factors," Nature cell biology, vol. 21, no. 1, pp. 102-112, 2019.

[13] S. A. Lambert, A. Jolma, L. F. Campitelli, P. K. Das, Y. Yin, M. Albu, X. Chen, J. Taipale, T. R. Hughes, and M. T. Weirauch, "The human transcription factors," Cell, vol. 172, no. 4, pp. 650-665, 2018.

[14] D. Kostrewa, M. E. Zeller, K.-J. Armache, M. Seizl, K. Leike, M. Thomm, and P. Cramer, "Rna polymerase ii-tfiib structure and mechanism of transcription initiation," Nature, vol. 462, no. 7271, pp. 323-330, 2009.

[15] G. Jenster, T. E. Spencer, M. M. Burcin, S. Y. Tsai, M.-J. Tsai, and B. W. O'Malley, "Steroid receptor induction of gene transcription: a two-step model," Proceedings of the National Academy of Sciences, vol. 94, no. 15, pp. 7879-7884, 1997.

[16] E. Kalkhoven, J. E. Valentine, D. M. Heery, and M. G. Parker, "Isoforms of steroid receptor co-activator 1 differ in their ability to potentiate transcription by the oestrogen receptor," The EMBO journal, vol. 17, no. 1, pp. 232-243, 1998.

[17] M. Beato, G. Chalepakis, M. Schauer, and E. P. Slater, "Dna regulatory elements for steroid hormones," Journal of steroid biochemistry, vol. 32, no. 5, pp. 737-747, 1989.

[18] J. A. Lefstin, J. R. Thomas, and K. R. Yamamoto, "Influence of a steroid receptor dna-binding domain on transcriptional regulatory functions." Genes $\& 6$ development, vol. 8, no. 23, pp. 2842-2856, 1994.

[19] L. A. Coons, S. C. Hewitt, A. B. Burkholder, D. P. McDonnell, and K. S. Korach, "Dna sequence constraints define functionally active steroid nuclear receptor binding sites in chromatin," Endocrinology, vol. 158, no. 10, pp. 3212-3234, 2017.

[20] C. Schaper, "Endogenous Binding of Steroid Molecules to DNA Nucleotides by a Ca2+/PO4- Process to Enable Gene Transcription," chemRxiv, 2020, https://doi.org/10.26434/chemrxiv.11868261.v1.

[21] T. A. Halgren, "Merck molecular force field. i. basis, form, scope, parameterization, and performance of mmff94," Journal of computational chemistry, vol. 17, no. 5-6, pp. 490-519, 1996.

[22] C. Schaper, "Competitive inhibition of cortisol by prostaglandins at the ligand binding domain of glucocorticoid receptors," bioRxiv, 2019, https://doi.org/10.1101/851501. 